\title{
An Introduction to The Modular Forensic Handwriting METHOD
}

In psychiatry, practitioners reach for what is affectionately known as the DSM (Diagnostic and Statistical Manual of Mental Disorders, now in its fifth edition) to reliably identify and diagnose aberrant social and behavioral conditions. The DSM is founded on extensive empirical research spanning many decades, adding credibility to its method of classifying complex emotional and neuropsychiatric states. Perhaps most importantly, the DSM is a living document, evolving in parallel with shifting human culture and insight gained from years of self-evaluation and criticism.

In many respects, the 2016 version of The Modular Forensic Handwriting Method (A Modular Approach) edited by Drs. Bryan Found and Carolyne Bird is the DSM equivalent for professional forensic handwriting examiners. The systematic modular approach developed by the editors attempts to standardize a human process. In the absence of unambiguous biomarkers, making an accurate diagnosis of a mental disorder requires a systematic process of elimination and inclusion of signs and symptoms. Practitioners of forensic feature-matching comparisons such as handwriting follow a similar process to determine whether an evidentiary sample is or is not consistent with a known source sample. Nonadherence with accepted practices can have significant human or probative consequences. The Modular Approach to forensic handwriting examination developed by Found and his colleagues transforms the tasks of elimination and inclusion into a systematic orderly process.

The 2016 version of A Modular Approach is an extensive update to the prior edition published in this journal in 1999. In the nearly two decades since the first edition was published, there has been significant research in the fields of motor control, forensics, human factors, and machine-learning with implications for handwriting examination. Citing recent research in the area of human factors, Found and Bird call attention to the potential for bias and the need to protect against "cognitive contamination of evidence."

\section{The Modular Approach}

Modules range from methods for establishing sufficiency of questioned and comparison samples through feature comparisons to interpretation and reporting. Within the seven modules devoted to feature comparison, the authors devote considerable attention to specific challenges facing the forensic document examiner including simulations, disguise, tracings, and risks to credible interpretation stemming from cognitive bias. The modules include several examples of handwriting to demonstrate the systematic process of feature extraction and comparison based on the presence of similar patterns, impressions, features, or characteristics in the sample and the source.

Forensic handwriting evidence has been criticized as lacking rigorous scientific validation. Indeed, as Found and Bird point out in the supplemental study included in this document, forensic handwriting examination grew out of practical application and not through the traditional scientific method of hypothesis development and testing. Existing empirical validation studies often have little bearing on actual casework. Conversely, the nature of casework with highly variable propositions and evidence does not lend itself to systematic study to validate a given approach or conclusion. Nevertheless, Found and Bird report findings from a multi-year study of over 27,000 opinions expressed by 28 document examiners designed to test the validity of their modular approach. Their research serves to motivate a standard approach to designing future validation studies that not only address prior criticisms of forensic handwriting examination but also strengthen the probative value of opinions expressed by trained examiners.

Michael P. Caligiuri, Ph.D.

Emeritus Professor

Department of Psychiatry

University of California, San Diego 